\title{
Eigenvectors of Matric Polynomials
}

\author{
Murray Mannos
}

\begin{abstract}
It is the object of this paper to compare the eigenvectors of an arbitrary $n \times n$ matrix $A$ over the complex field with those of the matric polynomia! $f(A)$. While it is well known that each eigenvector of $A$ is an eigenvector of $f(A)$, it is not, in general, true that $A$ and $f(A)$ have identical eigenvectors. In this regard a necessary and sufficient condition that $A$ and $f(A)$ have identical eigenvectors is given. The condition is that both (1) and (2) hold: (1) $f^{\prime}(\lambda) \neq 0$ for all eigenvalues $\lambda$ of the matrix $A$ corresponding to nonlinear elementary divisors.

(2) The values of $f(\mu)$ are distinct for all eigenvalues $\mu$ of the matrix $A$ corresponding to linear elementary divisors.

When either (1) or $(2)$ fails to hold, then $f(A)$ has eigenvectors that are not eigenvectors of A. This situation is also discussed.
\end{abstract}

The vector space of eigenvectors of the matrix $A$ corresponding to the eigenvalue $\lambda$ shall be denoted by $\Gamma_{\lambda}[A]$. The vector space spanned by the eigenvectors of $A$ shall be denoted by $V[A]$. Further, $d\left\{V_{\lambda}[A]\right\}$ and $d\{V[A]\}$ shall denote their dimensions. It is clear that each eigenvector of $A$ is an eigenvector of $f(A)$. That is, $V_{\lambda}[A] \subseteq V_{f(\lambda)}[f(A)]$ for each eigenvalue $\lambda$ of $A$. Thus $V[A] \subseteq V[f(A)]$.

Let $J$ be the Jordan canonical form of $A$. Then there exists a nonsingular matrix $P$ such that $P^{-1} A P=J$ and so $P^{-1} f(A) P=f(J)$.

Lemma 1. The eigenvectors of $A$ and $f(A)$ are identical if, and only if, the eigenvectors of $J$ and $f(J)$ are identrical.

Proof. This follows from the fact that $P \xi$ is an eigenvector of $P B P^{-1}$ if $\xi$ is an eigenvector of the matrix $B$.

Since $P\left\{\Gamma_{\lambda}[J]\right\}=V_{\lambda}[A]$, it follows that

$$
d\{V[f(A)]\}-d\{V[A]\}=d\{V[f(J)]\}-d\{V[J]\},
$$

where $P\left\{V_{\lambda}[J]\right\}$ denotes the space of all vectors $P \xi$, where $\xi$ is an eigenvector of $J$ corresponding to $\lambda$.

Lemma 2. If $D=\operatorname{diag}[\alpha, \alpha, \ldots ., \alpha ; \beta, \beta, \ldots, \beta$; $\ldots ; \pi, \pi, \ldots, \pi]$, where $\alpha, \beta, \ldots . . \pi$ are distinct, then $\Gamma[D]=V[f(D)]$. Furthermore, the eigenvectors of $D$ are identical with those of $f(D)$ if, and only if, $f(\alpha), f(\beta)$, .,$f(\pi)$ are all distinct.

Proof. If $D$ is of order $n$, it is clear that both the eigenvectors of $D$ and $f(D)$ each generate the whole $n$-dimensional vector space. If $f(\alpha)=f(\beta)$, then it is easily seen that $f(D)$ has eigenvectors corresponding to the eigenvalue $f(\alpha)=f(\beta)$, which are not eigenvectors of $D$.

Lemma 3.

$$
J_{m}(\lambda)=\left[\begin{array}{ccccccc}
\lambda & 1 & 0 & \ldots & 0 & 0 & 0 \\
0 & \lambda & 1 & \ldots & 0 & 0 & 0 \\
. & . & . & & . & . & . \\
. & . & . & & . & . & . \\
. & . & . & & . & . & . \\
0 & 0 & 0 & \ldots & 0 & \lambda & 1 \\
0 & 0 & 0 & \ldots & 0 & 0 & \lambda
\end{array}\right] \text {, }
$$

${ }^{1}$ Preparaicion of this paper was sponsored (in part) by the Office of Scientific Research, USAF.

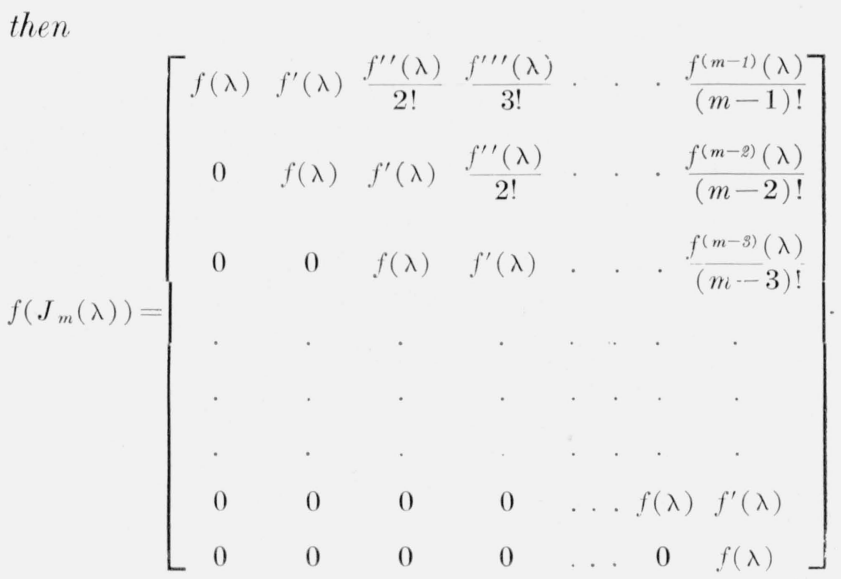

Proofs of the lemma may be found in Wedderburn ${ }^{2}$ and MacDuffee. ${ }^{3}$

Lemma 4. If $f^{\prime}(\lambda)=f^{\prime \prime}(\lambda)=\ldots=f^{(r)}(\lambda)=0$ and $f^{(r+1)}(\lambda) \neq 0(r=1,2, \ldots, m-2)$, then

$$
d\left\{V_{f(\lambda)}\left[f\left(J_{m}(\lambda)\right)\right]\right\}-d\left\{V_{\lambda}\left[J_{m}(\lambda)\right]\right\}=r,
$$

and conversely. Also,

$$
d\left\{V_{f(\lambda)}\left[f\left(J_{m}(\lambda)\right)\right]\right\}-d\left\{V_{\lambda}\left[J_{m}(\lambda)\right]\right\}=m-1
$$

if, and only if, $f^{\prime}(\lambda)=f^{\prime \prime}(\lambda)=\ldots=f^{m-1}(\lambda)=0$.

Proof. If $f^{\prime}(\lambda)=f^{\prime \prime}(\lambda)=\ldots=f^{(r)}(\lambda)=0$, but $f^{(r+1)}(\lambda) \neq 0$, it follows that the rank of the matrix $f\left(J_{m}(\lambda)\right)-f(\lambda) I$ is $m-(r+1)$, as may be observed by noting the nonzero minor of order $m-(r+1)$ in the upper right-hand corner of the matrix. Thus the nullity of $f\left(J_{m}(\lambda)\right)-f(\lambda) I$ is $r+1$. If the first $m-1$ derivatives of $f(x)$ vanish at $x=\lambda$, then the nullity of $f\left(J_{m}(\lambda)\right)-f(\lambda) I$ is $m$. In a similar fashion one finds that the matrix $J_{m}(\lambda)-\lambda I$ has nullity equal to 1 . This establishes the lemma.

Corollary. The matrices $J_{m}(\lambda)$ and $f\left(J_{m}(\lambda)\right)$ have identical eigenvectors if, and only if, $f^{\prime}(\lambda) \neq 0$.

It is to be noted that in this case $V_{f(\lambda)}\left[f\left(J_{m}(\lambda)\right)\right]$

2 J. H. M. Wedderburn, Lectures on Matrices, (Am. Math. Soc., Colloquium Pub. vol. 17, New York, N. Y., 1934).

${ }^{3}$ C. C. MacDuffee, An Introduction to Abstract Algebra (John Wiley \& Sons, 
and $\Gamma_{\lambda}\left[J_{m}(\lambda)\right]$ are each spanned by the single column vector $[1,0, \ldots, 0]^{T}$.

At this point it will be convenient to introduce the language of elementary divisors. Each block in the Jordan canonical form of a matrix $A$ corresponds to an elementary divisor $(x-\lambda)^{m}$, and conversely. Each such block is called the hypercompanion matrix $J_{m}(\lambda)$ of the polynomial $(x-\lambda)^{m}$.

Suppose the nonlinear elementary divisors of the matrix $A$ corresponding to the eigenvalue $\lambda$ are of the form $(x-\lambda)^{m_{\lambda}^{(1)}},(x-\lambda)^{m_{\lambda}^{(2)}}, \ldots,(x-\lambda)^{m_{\lambda}{ }^{\left(k_{\lambda}\right)}}$, where $m_{\lambda}^{(i)}\left(i=1,2, \ldots, k_{\lambda}\right)$ are integers such that $m_{\lambda}^{(1)}>m_{\lambda}^{(2)}>$. . $>m_{\lambda}^{\left(k_{\lambda}\right)}>1$. Furthermore, suppose that $(x-\lambda)^{m_{\lambda}^{(i)}}$ appears as an elementary divisor $n_{\lambda}^{(i)}$ times. Let $p_{\lambda}=\sum_{i=1}^{k_{\lambda}} n_{\lambda}^{(i)}$ denote the total number of nonlinear elementary divisors of $A$ corresponding to the eigenvalue $\lambda$. Denote by $n_{\lambda}$ the number of linear elementary divisors of $A$ corresponding to the eigenvalue $\lambda$. Further, denote by $K_{\lambda}$ the direct sum of the hypercompanion matrices of the elementary divisors corresponding to $\lambda$. Set

$$
d_{\lambda}=d\left\{V_{f(\lambda)}\left[f\left(K_{\lambda}\right)\right]\right\}-d\left\{V_{\lambda}\left[K_{\lambda}\right]\right\} .
$$

Lemma 5. If the first $m_{\lambda}^{(1)}-1$ derivatives of $f(x)$ vanish at $x=\lambda$, then $d_{\lambda}=\sum_{i=1}^{k \lambda} n_{\lambda}^{(i)}\left(m_{\lambda}^{(i)}-1\right)$; whereas, if the first $r<m_{\lambda}^{(1)}-1$ derivatives of $f(x)$ vanish at $x=\lambda$, but $f^{(r+1)}(\lambda) \neq 0$, then $d_{\lambda}=\sum_{i=1}^{l-1} n_{\lambda}^{(i)} r+\sum_{i=l}^{k_{\lambda}} n_{\lambda}^{(i)}\left(m_{\lambda}^{(i)}-1\right)$, where $m_{\lambda}^{(l)}$ is the largest of the integers $m_{\lambda}^{(i)}\left(i=1,2, \ldots, k_{\lambda}\right)$ such that $m_{\lambda}^{(l)} \leq r$.

Proof. This follows from lemma 4, the fact that the nullity of $K_{\lambda}-\lambda I$ is the sum of the nullities of the characteristic matrices of the hypercompanion matrices of the individual elementary divisors corresponding to the eigenvalue $\lambda$ and a similar statement about the nullity of $f\left(K_{\lambda}\right)-f(\lambda) I$.

Corollary. The matrices $K_{\lambda}$ and $f\left(K_{\lambda}\right)$ have identical eigenvectors if, and only if, $f^{\prime}(\lambda) \neq 0$.

Proof. This follows from the fact that $d_{\lambda}=0$.

The Jordan canonical form $J=\operatorname{diag}\left[K_{\alpha}, K_{\beta}, \ldots, K_{\pi}\right]$ of $A$ is a direct sum of matrices $K_{\lambda}$, where $\lambda=\alpha, \beta, \ldots$. runs through the distinct eigenvalues of $A$. The subsequent theorems of this paper involve the following main conditions:

Condition 1. $f^{\prime}(\lambda) \neq 0$ for all eigenvalues $\lambda$ of the matrix A corresponding to nonlinear elementary divisors.

Condition 2. The values $f(\mu)$ are distinct for all eigenvalues $\mu$ of the matrix $A$ corresponding to linear elementary divisors.

The first theorem concerns the case where condition 1 does not hold, whether condition 2 holds or not.

Theorem 1. If condition 1 does not hold, then

$$
d\{V[f(A)]\}-d\{V[A]\}=\sum_{\lambda} d_{\lambda},
$$

where $\lambda$ varies through all distinct eigenvalues of $A$ corresponding to nonlinear elementary divisors, and $d_{\lambda}$ is computed as in lemma 5.

Proof. One notes first that

and

$$
V[J]=V_{\alpha}[J]+V_{\beta}[J]+\ldots+V_{\pi}[J],
$$

$$
\Gamma[f(J)]=\Gamma_{f(\alpha)}[f(J)]+\Gamma_{f(\beta)}[f(J)]+\ldots+\Gamma_{f(\pi)}[f(J)] .
$$

If $f(\alpha), f(\beta), \ldots, f(\pi)$ are distinct, for a fixed eigenvalue $\lambda$, the nullity of $J-\lambda I$ is the same as the nullity of $K_{\lambda}-\lambda I$, and the nullity of $f(J)-f(\lambda) I$ is the same as the nullity of $f\left(K_{\lambda}\right)-f(\lambda) I$. It follows that

$$
d\left\{V\left[_{f(\lambda)}[f(J)]\right\}-d\left\{\Gamma_{\lambda}[J]\right\}=d_{\lambda} .\right.
$$

Summing over distinct eigenvalues, one obtains

$$
d\{V[f(J)]\}-d\{T[J]\}=\sum_{\lambda} d_{\lambda} .
$$

By the statement following the proof of lemma 1 it follows that

$$
d\{V[f(A)]\}-d\{V[A]\}=\sum_{\lambda} d_{\lambda} .
$$

If $f(\alpha)=f(\beta)=\ldots=f(\rho)$, then the nullity of $f(J)-f(\alpha) I$ minus the sum of the nullities of $J-\alpha I$, $J-\beta I, \ldots$, and $J-\rho I$ is $d_{\alpha}+d_{\beta}+\ldots+d_{\rho}$ and

$$
\begin{array}{r}
d\left\{V_{f(\alpha)}[f(J)]\right\}-d\left\{V_{\alpha}[J]\right\}-d\left\{\Gamma_{\beta}[J]\right\}-\ldots-d\left\{V_{\rho}[J]\right\} \\
=d_{\alpha}+d_{\beta}+\ldots+d_{\rho} .
\end{array}
$$

Summing, one obtains in either case

$$
d\{V[f(A)]\}-d\{V[A]\}=\sum_{\lambda} d_{\lambda},
$$

where $\lambda$ runs through all the distinct eigenvalues of $A$ corresponding to nonlinear elementary divisors.

The next theorem concerns the case in which condition 1 holds but condition 2 does not.

Theorem 2. If condition 1 holds, than $V[A]=V[f(A)]$. In this case $\sum_{\lambda} d_{\lambda}=0$, where $\lambda$ varies over all distinct eigenvalues corresponding to nonlinear elementary divisors.

If $f(\alpha)=f(\beta)=\ldots=f(\rho)$, suppose that

$d\left\{V_{f(\alpha)}[f(J)]\right\}=d\left\{V_{\alpha}[J]\right\}$

$$
+d\left\{\Gamma_{\beta}[J]\right\}+\ldots+d\left\{I_{\rho}[J]\right\}=s .
$$

Then as in lemma 2 the $s$-dimensional space $V_{\alpha}[J]+V_{\beta}[J]+\ldots+V_{\rho}[J]$ contains vectors that are not eigenvectors of $J$, but $V_{f(\alpha)}[f(J)]$ consists solely of eigenvectors of $f(\mathcal{J})$ corresponding to $f(\alpha)$.

Corollary. If $f(\alpha)=f(\beta)=\ldots=f(\rho)$, then

$$
d\left\{V_{f(\alpha)}[f(A)]\right\}-d\left\{V_{\alpha}[A]\right\}=\sum_{\lambda} d_{\lambda}+\left(p_{\lambda}+n_{\lambda}\right),
$$

where $\lambda$ runs over all eigenvalues among $\alpha, \beta, \ldots, \rho$ which correspond to nonlinear elementary divisors and 
where $p_{\lambda}+n_{\lambda}$ is the total number of elementary divisors (nonlinear and linear) corresponding to $\lambda$.

Finally, the next result covers the case in which conditions 1 and 2 both hold.

Theorem 3. The matrices $A$ and $f(A)$ have identical eigenvectors if, and only if (1) $f^{\prime}(\lambda) \neq 0$ for all eigenvalues $\lambda$ of the matrix $A$ corresponding to nonlinear elementary divisors, and (2) the values $f(\mu)$ are distinct for all eigenvalues $\mu$ of the matrix A corresponding to linear elementary divisors.

Remark 1. It may readily be seen that $V[J]$ and $V[f(J)]$ each can be generated by a set of linearly independent vectors, each of which has a 1 in a single component and 0 in all the remaining components. Thus if $J=P^{-1} A P$ is the Jordan canonical form of $A$, than $V[f(A)]$ can be generated by a subset of the column vectors of $P$.

Remark 2. A simple application of the foregoing theory shows that if $A$ is a $2 \times 2$ matrix, then $A$ and $f(A)$ have the same eigenvectors unless either (1) $A$ is diagonable and has distinct eigenvalues $\alpha, \beta$ for which $f(\alpha)=f(\beta)$, or (2) $A$ is nondiagonable and $f(x)=k(x)(x-\alpha)^{2}+c$, where $\alpha$ is the eigenvalue of $A$, $k(x)$ is an arbitrary polynomial, and $c$ is an arbitrary constant.

The following example is given as an illustration:

$$
\text { Let } A=\left[\begin{array}{rrrrrrr}
1 & 0 & 0 & 0 & 0 & 0 & 0 \\
0 & 0 & 1 & 0 & 0 & 0 & 0 \\
0 & 0 & 0 & 1 & 0 & 0 & 0 \\
0 & 0 & 0 & 0 & 0 & 0 & 0 \\
0 & 0 & 0 & 0 & 0 & 0 & 0 \\
-1 & -1 & -1 & -1 & -1 & -2 & -1 \\
1 & 0 & 1 & 1 & 0 & -1 & 0
\end{array}\right]
$$

and $f(x)=x^{6}+x^{5}-x^{4}-x^{3}+2$.

$$
\text { For } P=\left[\begin{array}{lllllll}
1 & 0 & 0 & 0 & 0 & 0 & 0 \\
0 & 1 & 0 & 0 & 0 & 0 & 0 \\
0 & 0 & 1 & 0 & 0 & 0 & 0 \\
0 & 0 & 0 & 1 & 0 & 0 & 0 \\
0 & 0 & 0 & 0 & 1 & 0 & 0 \\
0 & 0 & 0 & 0 & 0 & 1 & 0 \\
1 & 1 & 1 & 1 & 1 & 1 & 1
\end{array}\right]
$$

it may be verified that

$$
P^{-1}=\left[\begin{array}{rrrrrrr}
1 & 0 & 0 & 0 & 0 & 0 & 0 \\
0 & 1 & 0 & 0 & 0 & 0 & 0 \\
0 & 0 & 1 & 0 & 0 & 0 & 0 \\
0 & 0 & 0 & 0 & 1 & 0 & 0 \\
0 & 0 & 0 & 0 & 0 & 1 & 0 \\
-1 & -1 & -1 & -1 & -1 & -1 & 1
\end{array}\right]
$$

and that the Jordan canonical form of $A$ is

$$
J=P^{-1} A P=\left[\begin{array}{rrrrrrr}
1 & 0 & 0 & 0 & 0 & 0 & 0 \\
0 & 0 & 1 & 0 & 0 & 0 & 0 \\
0 & 0 & 0 & 1 & 0 & 0 & 0 \\
0 & 0 & 0 & 0 & 0 & 0 & 0 \\
0 & 0 & 0 & 0 & 0 & 0 & 0 \\
0 & 0 & 0 & 0 & 0 & -1 & 1 \\
0 & 0 & 0 & 0 & 0 & 0 & -1
\end{array}\right],
$$

where

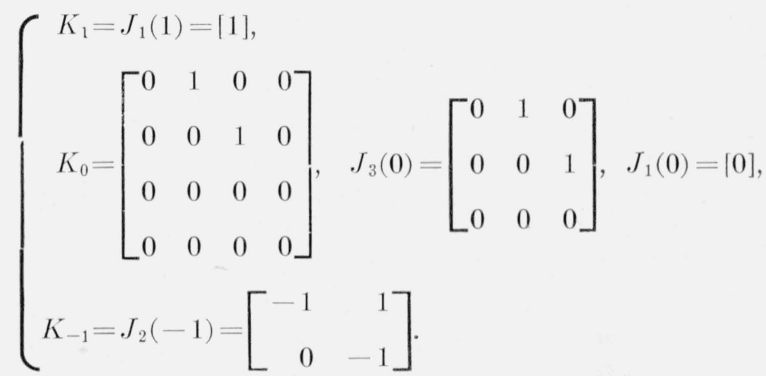

Since

$$
\left\{\begin{array}{l}
f(0)=f(1)=f(-1)=2, \\
f^{\prime}(0)=f^{\prime}(-1)=0, \quad f^{\prime}(1) \neq 0, \\
f^{\prime \prime}(0)=0,
\end{array}\right.
$$

it follows that

$$
f(J)=J^{6}+J^{5}-J^{4}-J^{3}+2 I=\left[\begin{array}{ccccccc}
2 & 0 & 0 & 0 & 0 & 0 & 0 \\
0 & 2 & 0 & 0 & 0 & 0 & 0 \\
0 & 0 & 2 & 0 & 0 & 0 & 0 \\
0 & 0 & 0 & 2 & 0 & 0 & 0 \\
0 & 0 & 0 & 0 & 2 & 0 & 0 \\
0 & 0 & 0 & 0 & 0 & 2 & 0 \\
0 & 0 & 0 & 0 & 0 & 0 & 2
\end{array}\right]
$$

It is readily seen that $V_{1}[J]$ is generated by $[1,0,0,0,0,0,0]^{T}, \mathrm{~V}_{0}[J]$ is generated by $[0,1,0,0,0,0,0]^{T}$ and $[0,0,0,0,1,0,0]^{T}$, and $\mathrm{V}_{-1}[J]$ is generated by $[0,0,0,0,0,1,0]^{T}$. Since $V[J]=V_{1}[J]+V_{0}[J]+V_{-1}[J]$, it follows that $d\{V[J]\}=4$. Since $f[J]$ is a scalar matrix, $d\{V[f(J)]\}=7$. Hence $d\{[f(A)]\}-d\{V[A]\}=3$. The same result is arrived at by the use of theorem 1 , where $\sum_{\lambda} d_{\lambda}$ is calculated as in lemma 5 . Since $f^{\prime}(0)=0, f^{\prime \prime}(0)=0$, and $f^{\prime}(-1)=0$, where $0,-1$ are the eigenvalues corresponding to nonlinear elementary divisors, it follows that

and

$$
d_{0}=n_{0}^{(1)}\left(m_{0}^{(1)}-1\right)=1 \cdot 2=2
$$

$$
d_{-1}=n_{1}^{(1)} r=1 \cdot 1=1
$$


and

$$
\sum_{\lambda} d_{\lambda}=d_{0}+d_{-1}=2+1=3,
$$

as before. By the corollary following theorem 2,

$$
\begin{aligned}
d\left\{V_{2}[f(A)]\right\}-d\left\{V_{1}[A]\right\} & =d_{0}+d_{-1}+p_{0}+p_{-1}+n_{0}+n_{-1} \\
& =2+1+1+1+1+0=6,
\end{aligned}
$$

which checks with the observed results above.
From a glance at the above eigenvectors generating $V[J]$, it is clear that $V[A]$ is generated by the first, second, fifth, and sixth-column vectors of $P$. Since $f(A)$ is a scalar, $V[f(A)]$ is generated by all seven column vectors of $P$.

The author thanks A. H. Clifford, O. Taussky, and N. Wiegmann for their helpful suggestions.

Washington, December 1, 1952. 